\title{
ARTICLE
}

\section{Mediator MED23 cooperates with RUNX2 to drive osteoblast differentiation and bone development}

\author{
Zhen Liu', Xiao Yao ${ }^{1}$, Guang Yan${ }^{1}$, YiChi Xu² ${ }^{2}$ Jun Yan², Weiguo Zou ${ }^{1} \&$ Gang Wang ${ }^{1}$
}

How lineage specifiers are regulated during development is an outstanding question, and the molecular regulation of osteogenic factor RUNX2 remains to be fully understood. Here we report that the Mediator subunit MED23 cooperates with RUNX2 to regulate osteoblast differentiation and bone development. Med23 deletion in mesenchymal stem cells or osteoblast precursors results in multiple bone defects similar to those observed in Run $\times 2^{+/-}$mice. In vitro, Med23-deficient progenitor cells are refractory to osteoblast differentiation, and Med23 deficiency reduces Runx2-target gene activity without changing Runx2 expression. Mechanistically, MED23 binds to RUNX2 and modulates its transcriptional activity. Moreover, Med23 deficiency in osteoprogenitor cells exacerbates the skeletal abnormalities observed in Runx $2^{+/-}$mice. Collectively, our results establish a genetic and physical interaction between RUNX2 and MED23, suggesting that MED23 constitutes a molecular node in the regulatory network of anabolic bone formation and related diseases.

\footnotetext{
${ }^{1}$ State Key Laboratory of Cell Biology, CAS Center for Excellence in Molecular Cell Science, Institute of Biochemistry and Cell Biology, Shanghai Institutes for Biological Sciences, Chinese Academy of Sciences, 320 Yueyang Road, Shanghai 200031, China. ${ }^{2}$ CAS-MAP Partner Institute for Computational Biology, Shanghai Institutes for Biological Sciences, Chinese Academy of Sciences, 320 Yueyang Road, Shanghai 200031, China. Correspondence and requests for materials should be addressed to W.Z. (email: zouwg94@sibcb.ac.cn) or to G.W. (email: gwang@sibcb.ac.cn).
} 
$\mathrm{M}$ esenchymal stem cells (MSCs) are multipotent progenitors that can self-renew and differentiate into several distinct cell lineages, including osteoblasts, chondrocytes and adipocytes ${ }^{1,2}$. Their differential potentials and intrinsic properties make MSCs of great interest for applications in a variety of cell-based therapies ${ }^{3-6}$. In the past few decades, several master regulators that specify the various cell fates associated with MSCs have been identified: for example, Runx2 triggers MSCs to differentiate into osteoblasts ${ }^{7,8}$. The function of Runx2 in osteoblast differentiation was initially demonstrated by observations that Runx $2^{-1-}$ mice lack both mature osteoblasts and a mineralized skeleton ${ }^{8-10}$. Although Run $\times 2^{+I-}$ mice do undergo skeletal mineralization, the process is impaired, with delayed closure of the fontanelles and clavicular hypoplasia ${ }^{10}$, resembling the cleidocranial dysplasia (CCD) phenotype that arises from Runx2 haploinsufficiency in

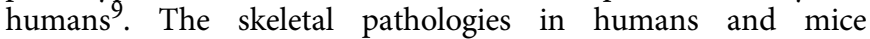
highlight the importance of the precise regulation of Runx2 activity during osteogenesis.

Apart from these key transcription factors, several cofactors have been found to be involved in the fine tuning of cell-fate determination ${ }^{11-13}$. One such cofactor is the transcriptional complex Mediator, which is a multiprotein complex that has been evolutionarily conserved from yeast to metazoans. The Mediator complex is recruited by transcription factors in response to developmental or environmental signals. Through direct protein interactions, Mediator links transcription factors to the RNA polymerase II (Pol II) machinery and modulates the transcriptional output ${ }^{14}$.

Our previous research demonstrated that the Mediator MED23 subunit acts as a molecular modulator to control the balance between adipogenesis and myogenesis ${ }^{15,16}$. However, it remained unknown whether MED23 is also involved in the development of osteoblasts, which, like adipocytes and myocytes, are also derived from mesenchymal progenitors. In this study, we provide evidence of a role for MED23 in osteogenesis. Mice deficient in Med23 in MSCs or preosteoblasts display defective bone formation and impaired osteoblast differentiation, resembling the skeletal phenotype of Runx2 $2^{+/}$mice. Further molecular studies demonstrate that MED23 cooperatively interacts with RUNX2 in the regulation of osteogenesis. Our results reveal that MED23 is an important regulator of osteogenesis and is essential for the transcriptional activity of RUNX2.

\section{Results}

Med23 deficiency in MSCs impaired bone ossification. To investigate the role of Med23 in the commitment of MSCs to an osteoblast fate, we generated a $\mathrm{Med} 23$ conditional knockout mouse model by crossing Med23 $3^{f l / f l}$ mice $^{17}$ with Prx1-Cre mice (Fig. 1a). The latter is a transgenic line wherein Cre activity is largely confined to ventral and craniofacial mesenchyme ${ }^{18,19}$. Western blot assays verified that MED23 was largely abrogated in bone marrow-derived MSCs (Fig. 1b). MSC-specific Med23 deletion had no effect on survival or fertility. However, Med23 $3^{f / f l}$; Prx1-Cre mice (Med23 ${ }_{M S C}{ }^{-/}$) were shorter and underweight relative to their control littermates. Furthermore, as the Med $23_{M S C}{ }^{-1-}$ mice aged, they developed a marked dwarfism phenotype with short legs that was independent of sex (Fig. 1c,d). The heterozygous mice $\left(\operatorname{Med} 23_{\mathrm{MSC}^{+}}\right)$appear normal (Supplementary Fig. 1d). Consistent with this observation, the level of MED23 was barely reduced in bone tissue samples from Med $23_{\mathrm{MSC}^{+}} /-$mice (Supplementary Fig. 1c).

Notably, the membranous ossification of the skull was impaired in $\mathrm{Med} 23_{M S C}{ }^{-/-}$newborns compared with control littermates. The anterior and posterior fontanelles were enlarged and the cranial sutures were wider in $M e d 23_{M S C}{ }^{-/}$mice. Defective osteogenesis was not limited to cranial tissues, as the clavicles became thinner and shorter, and the sternum xiphoid was also hypoplastic (Fig. 1e). And these defects occurred as early as E15.5 when the bone mass is formed (Supplementary Fig. 1a). By contrast, a skeletal analysis of $M e d 23_{M S C}+/-$ mice confirmed that they were phenotypically normal relative to control littermates (Supplementary Fig. 1b).

To further determine the function of MED23 in the skeletal system, we utilized micro-quantitative computed tomography micro computed tomography $(\mu-\mathrm{CT})$ to compare skeletal elements isolated from $\mathrm{Med} 23_{M S C^{-/-}}$mice with corresponding elements from control littermates. As shown in Fig. 1f, 6-day-old Med $23_{M S C}{ }^{-1-}$ mice displayed hypomineralization of the calvaria compared with control mice. In addition, we found that 1-monthold Med $23_{M S C}{ }^{-1-}$ mice were osteopenic, with reduced bone mineral density (BMD) and bone volume per tissue volume) (BV/TV) in the femoral trabecular bone relative to age-matched control littermates. Further analysis showed that the reduced trabecular number (Tb.N) of Med $23_{M S C}{ }^{-1-}$ mice was accompanied by decreased trabecular thickness ( $\mathrm{Tb}$. Th) and a reduction in the Tb.N compared with those of control mice (Fig. 1g,h). Consistent with the decreased BMD in Med23 ${ }_{M S C}^{-/-}$mice, an ELISA assay of PINP (N-terminal propeptide of type I procollagen), a marker of bone formation, revealed a reduced bone formation rate in $M e d 23_{M S C}{ }^{-1-}$ mice (Fig. 1i). In adults, bone is continuously remodelled by bone-resorbing osteoclasts and bone-forming osteoblasts ${ }^{20}$. We performed a histological analysis and in vitro osteoclastogenesis experiment to exclude the possibility that the decreased bone density in the long bones of Med $23_{M S C}{ }^{-1-}$ mice could be attributed to increased osteoclast differentiation and/or activity. We found that osteoclast activity was comparable between $\mathrm{Med} 23_{\mathrm{MSC}}{ }^{-1-}$ mice and their control littermates (Supplementary Fig. 2a,b). Moreover, the expression of osteoclastic genes did not change either (Supplementary Fig. 2c). Taken together, these results suggest an important role for Med 23 in bone formation.

Ablation of Med23 in preosteoblasts reduced bone formation. To further determine whether the abnormal osteogenesis in Med $23_{M S C}{ }^{-1-}$ mice results from a primary defect in osteoblast development, we generated an osteoblast-specific Med23-deleted mouse model (Med23 $3_{o b}-1-$ mice) by crossing Med $23^{f l f l}$ mice with osterix $(O s x)$-Cre mice, a line in which Cre expression is primarily restricted to osteoblast precursors ${ }^{21}$ (Fig. 2a). Western blot assays showed that MED23 decreased in the bone of both $M e d 23_{\mathrm{ob}}+1-$ and $\mathrm{Med} 23_{\mathrm{ob}}{ }^{-1-}$ mice (Supplementary Fig. 3b). Consistent with decline in protein of MED23, histological analysis by alcian blue and alizarin red (ARS) staining showed defects in the skeleton occurring in both $\mathrm{Med} 23_{\mathrm{ob}}{ }^{+/-}$and $\mathrm{Med} 23_{\mathrm{ob}}{ }^{-I-}$ newborns, and more severe in the latter, including abnormality in calvarial and clavicle ossification (Supplementary Fig. 3a). However, Os $x$-Cre transgenic mice have been found to manifest defects in bone phenotype, such as to delay bone mineralization and develop scapula calluses ${ }^{22,23}$. To exclude such effect of Osx-Cre, we made a comparison between $M e d 23_{\mathrm{ob}}-I-$ mice and $M e d 23_{\mathrm{ob}}+I-$ control littermates that were both in the context of Osx-Cre. Again, Med23ob ${ }^{-/-}$mice developed runty and underweight compared with age-matched control littermates (Fig. 2b,c). Mice with $\mathrm{Med} 23$ deficiency in osteoblast reproduced the phenotype of $\mathrm{Med} 23_{\mathrm{MSC}}^{-/-}$mice, including impaired membranous ossification of calvarial bones and dysplasia of the clavicles, although the sternum appeared normal. In the $\operatorname{Med} 23_{o b}{ }^{-1-}$ mice, the bone was obviously porous; this feature was more evident in the bones of the skull (Fig. 2d,e). $\mu \mathrm{CT}$ analysis 
a

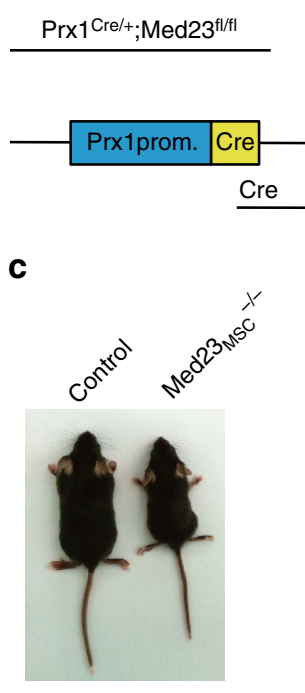

f

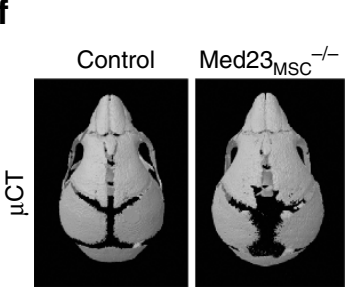

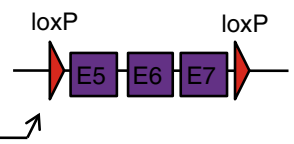

d

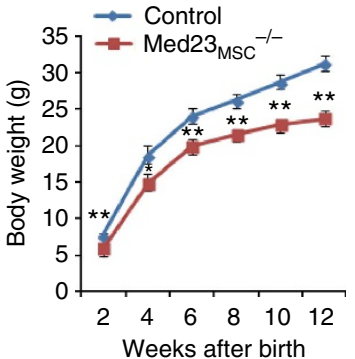

b

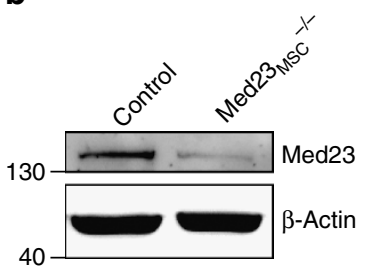

e

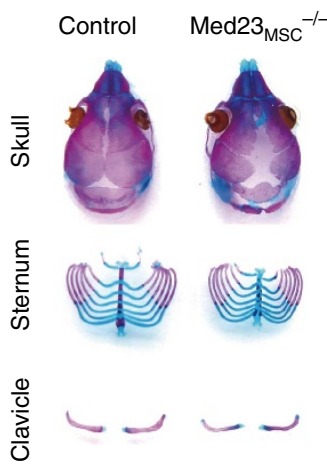

i

g
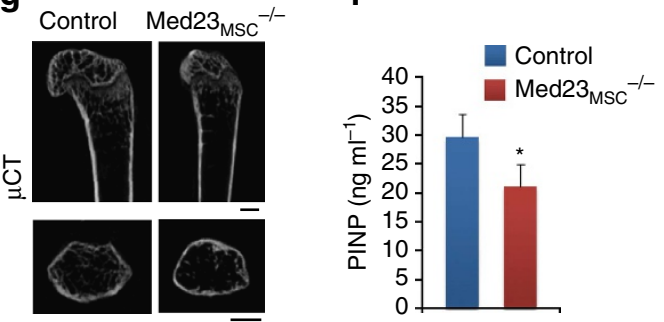

h
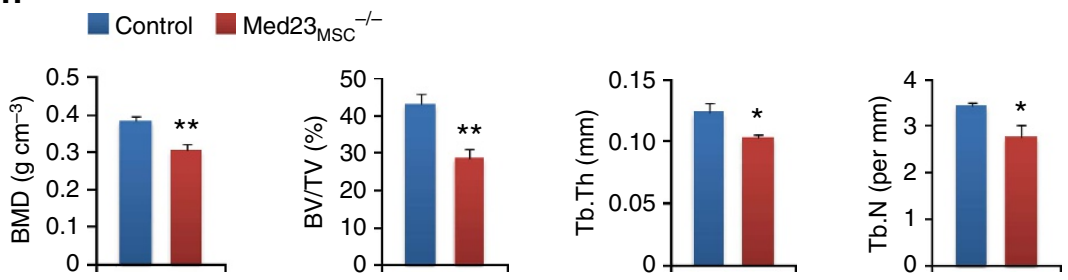

Figure 1 | Inactivation of $\mathbf{M e d} 23$ in mesenchymal stem cells causes impaired bone ossification. (a) Illustration of Med23 deletion in Prx1-expressing mesenchymal stem cells. (b) Expression of Med23 protein in bone marrow-derived mesenchymal stem cells from 6- to 8-week-old control (Med23 $3^{\mathrm{fl} / \mathrm{fl})}$ and Med23 $3_{\mathrm{MSC}}-/$ - mice. (c) Representative view of 1-month-old control and Med23 $\mathrm{MSC}^{-1-}$ mice. (d) Body weight of male control mice and

Med23 $\mathrm{MSC}^{-/-}$littermates measured at different age points ( $n=3$ for each group, $t$-test). Data represent means \pm s.d. ${ }^{\star} P<0.05,{ }^{\star \star} P<0.01$. (e) Skeletal preparations from control and $M e d 23_{\mathrm{MSC}^{-}}-$newborns were double stained with Alizarin red and Alcian blue. (f) Microcomputed tomography ( $\mu \mathrm{CT}$ ) image of skulls from control and Med23 $\mathrm{MsC}^{-1-}$ mice at postnatal day 6 (P6). (g) $\mu \mathrm{CT}$ images of distal femurs from 1-month-old control and Med23 $\mathrm{MsC}^{-1-}$ mice (top panel, longitudinal view; bottom panel, axial view of the metaphyseal region). Scale bar, $1 \mathrm{~mm}$. (h) Quantitative $\mu \mathrm{CT}$ analysis of distal femurs from 1-month-old control $(n=5)$ and $\operatorname{Med} 23_{\mathrm{MSC}}{ }^{-/-}$mice $(n=4)$, including bone mass density (BMD), bone volume per tissue volume (BV/TV), trabecular thickness (Tb.Th) and trabecular number (Tb.N). Data represent means \pm s.e.m. $t$-test, ${ }^{\star} P<0.05,{ }^{\star \star} P<0.01$. (i) ELISA analysis of serum PINP $\left(\mathrm{ng} \mathrm{ml}^{-1}\right)$ from 1-month-old control and Med23 $\mathrm{MSC}^{-/-}$mice $(n=4)$. Data represent means \pm s.d. $t$-test, ${ }^{\star} P<0.05,{ }^{\star \star} P<0.01$.

further confirmed the osteopenic phenotype of $\mathrm{Med} 23_{o b}-/-$ mice (Fig. 2f,g). Likewise, relatively lower levels of serum PINP indicated a decreased bone formation rate in $M e d 23_{o b}-1-$ mice (Fig. 2h). Again, in vivo and in vitro osteoclastogenesis showed comparable levels of osteoclasts between $M e d 23_{o b}-/-$ mice and control littermates (Supplementary Fig. $4 \mathrm{a}-\mathrm{c}$ ). Hence, the $\mathrm{Med} 23_{o b}{ }^{-1-}$ mice recapitulate the defects observed in $\mathrm{Med} 23_{\mathrm{MSC}}{ }^{-1-}$ mice with striking fidelity, supporting the conclusion that $\mathrm{Med} 23$ is necessary to the differentiation and function of committed osteoblast precursors.

Med23 is required for the osteoblast differentiation of MSCs. Next, we asked whether Med23 deficiency affects the osteogenic potential of MSCs in a cell-autonomous manner. Primary MSCs were isolated from the bone marrow of control and Med $23_{\mathrm{MSC}}{ }^{-1}$ - littermates, and the deficiency of Med23 in the MSCs from Med23 $3_{\text {MSC }}^{-I}$ mice was confirmed by western blot assay (Fig. 3a). Med23 deficiency in MSCs does not appear to alter the cell viability or growth rate, indicated by comparable numbers of colony-forming unit fibroblasts (c.f.u.-F) (Fig. 3b). However, Med23-I- MSCs displayed markedly decreased alkaline phosphatase (ALP) activity and mineralization (Fig. 3c,d). In addition, the mRNA levels of osteogenic genes, such as osteocalcin (Ocn), were significantly downregulated

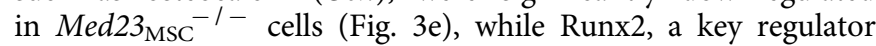
in bone development, did not change during osteogenesis 
a

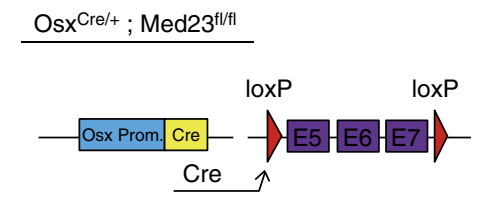

d

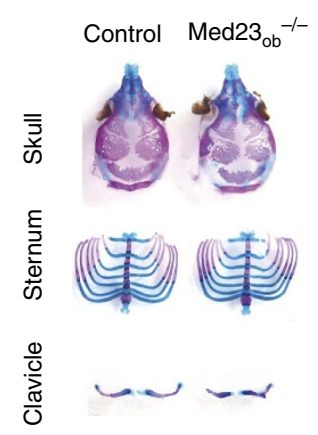

g

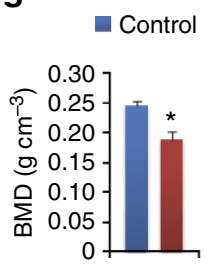

b

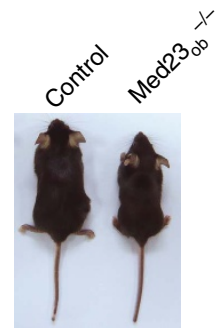

e

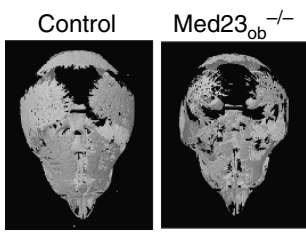

c

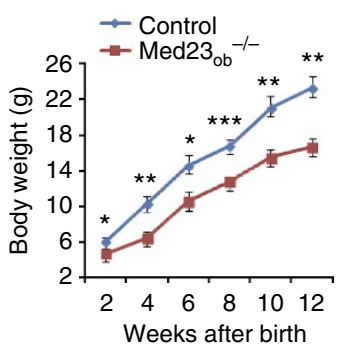

f

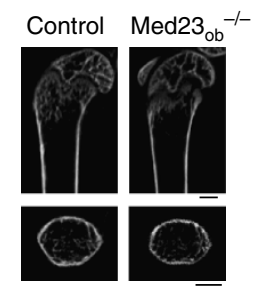

h

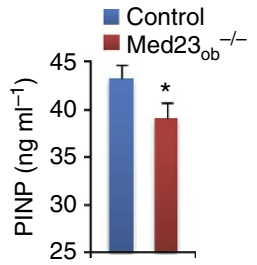

Figure 2 | Inactivation of Med23 in osteoblast progenitors results in defects in bone formation. (a) Illustration of Med23 deletion in

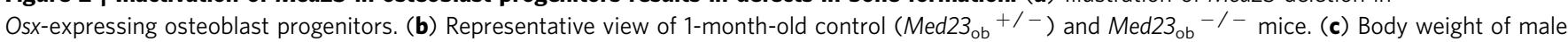
control mice and Med23 ${ }_{\mathrm{ob}}-/$ - littermates measured at different age points $(n=3)$. Data represent means \pm s.d. $t$-test, ${ }^{\star} P<0.05,{ }^{\star \star} P<0.01,{ }^{\star \star \star} P<0.001$. (d) Skeletal preparations from control and $M e d 23_{\mathrm{ob}}-/-$ newborns were double stained with Alizarin red and Alcian blue. (e) $\mu \mathrm{CT}$ image of skulls from control and Med23 $3_{\mathrm{ob}}{ }^{-/-}$mice at postnatal day 6 (P6). (f) $\mu \mathrm{CT}$ images of distal femurs from 1-month-old control and Med23 ${ }_{\mathrm{ob}}-/-$ mice. Scale bar, $1 \mathrm{~mm}$. (g) Quantitative $\mu \mathrm{CT}$ analysis of trabecular bone in the distal femurs from 1-month-old control and Med23 $3_{\mathrm{ob}}-/-$ mice $(n=3)$. Data represent means \pm s.e.m. $t$-test, ${ }^{\star} P<0.05$. (h) ELISA analysis of serum PINP ( $\mathrm{ng} \mathrm{ml}{ }^{-1}$ ) from 1-month-old control and Med23 $3_{\mathrm{ob}}{ }^{-/-}$mice $(n=4)$. Data represent means \pm s.d. $t$-test, ${ }^{\star} P<0.05$.

(Fig. 3a,e). Similarly, impaired osteogenesis was observed in Med23-deficient osteoblasts too (Supplementary Fig. 6). These results indicated that $\mathrm{Med} 23$ functioned necessarily in osteogenesis in vitro.

To exclude developmental differences between control and $M e d 23^{-I-}$ primary MSCs, we infected wild-type primary bone marrow MSCs with retroviruses expressing either shRNAs targeting Med23 or control shRNA. Consistent with the differentiation difference between WT and Med23 KO primary MSCs, shRNA-mediated acute knockdown of Med23 in MSCs also resulted in decreased osteogenesis, as indicated by ALP and ARS staining (Supplementary Fig. 5a). Real-time PCR analysis showed that Med23 knockdown reduced the expression of osteogenic marker genes such as Osx, Alp, collagen type I (Colla1) and Ocn. However, noticeably, the expression of Runx2, the master regulator for osteoblast differentiation, was unaffected by $M e d 23$ deficiency (Supplementary Fig. 5b).

Collectively, these results showed that $\operatorname{Med} 23$ is required for osteoblast differentiation. The key osteogenic genes downregulated in Med23-deficient cells are known targets of Runx2, suggesting that MED23 might regulate osteoblast differentiation via Runx2.

Med23 regulates the gene network of bone development. Despite our observation that the expression of Runx2, a master transcription factor for osteoblast differentiation, was not affected by $\mathrm{Med} 23$ deletion during osteogenesis, $\mathrm{Med} 23_{M S C}{ }^{-/-}$mice appeared to phenocopy the skeletal defects of Runx $2^{+/-}$mice, including delayed closure of the fontanelles, clavicular hypoplasia and decreased bone density ${ }^{10}$, suggesting that Med23 and Runx2 share a functional mechanism. To compare the effects of Med23 and Runx2 on global gene expression patterns, we performed RNA-seq with total RNA extracted from the calvaria of control and Med23-1- littermates, as well as WT and Runx $2^{+/-}$ littermates. Globally, Med23 $\mathrm{MSC}^{-1-}$ versus control and Runx $2^{+/}$ - versus WT revealed a significantly positive correlation in global gene expression (Fig. 4a). We next found that downregulated genes in $\mathrm{Med} 23_{\mathrm{MSC}}{ }^{-/-}$set presented in a similar pattern in Runx $2+1-$ set (Fig. 4b). In more details, 438 genes were downregulated $>1.5$-fold in the $\mathrm{Med} 23_{\mathrm{MSC}}{ }^{-1-}$ set compared with the corresponding control, and 504 genes were downregulated $>1.5$-fold in the Run $\times 2^{+1-}$ set compared with the control and a total of 124 genes overlapped between the 2 groups (Fig. 4c). These data further implied that these two molecules may function cooperatively. We then performed a gene ontology (GO) analysis of the set of 1.5-fold significantly downregulated genes from the $\mathrm{Med} 23_{\mathrm{MSC}}{ }^{-1-}$ sample. The results showed that these genes were enriched for associations with bone development and ossification (Fig. 4d). We verified the expression of osteoblast-specific genes in the calvaria from control and Med23 $3_{\mathrm{MSC}}{ }^{-1}$ newborns by real-time PCR and found that osteogenic genes such as Osx, Alp, Colla1 and Ocn 
a

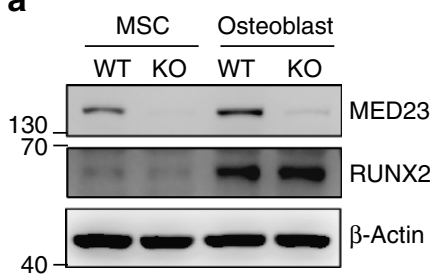

C

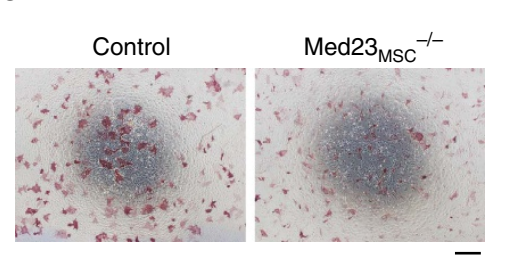

b

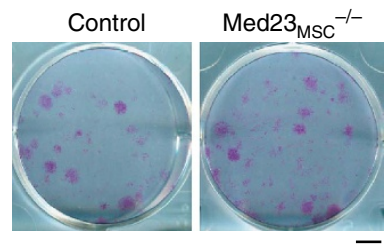

d
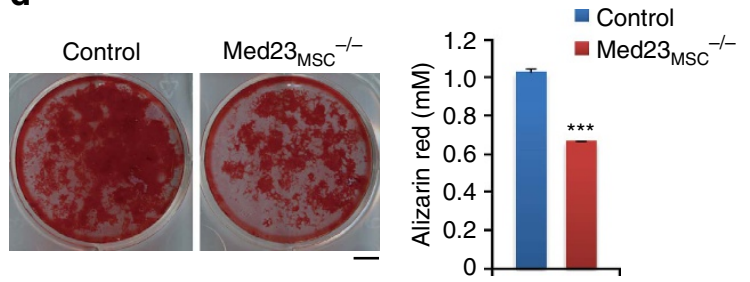

e

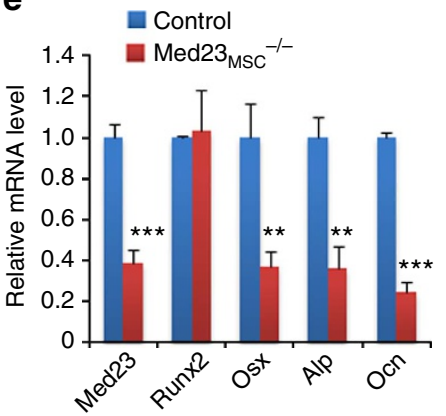

Figure 3 | Med23 deficiency inhibits osteoblast differentiation. (a) Expression of Med23 and Runx2 in bone marrow-derived mesenchymal stem cells and calvarial osteoblasts from 6 to 8 -week-old control and Med23 $\mathrm{MSC}^{-/}-$mice. Isolated cells were expanded and analysed by western blotting. (b) c.f.u.-F assay for bone marrow cells from control and Med23 $\mathrm{MSC}^{-/}$- littermates. Representative images of c.f.u.-Fs stained with crystal violet (left, scale bar, $0.5 \mathrm{~cm}$ ) and quantification of c.f.u.-Fs (right, $n=3$ for each group.). Data represent means \pm s.d. $t$-test. (c) ALP staining of bone marrow mesenchymal stem cells after cultured for 7 days in osteogenic medium. Scale bar, $500 \mu m$. (d) c.f.u.-ob assay for bone marrow of control and Med23 $\mathrm{MSC}^{-/-}$littermates. Bone marrow cells were cultured in osteogenic medium for 21 days, followed by staining with Alizarin red (left, scale bar, $0.5 \mathrm{~cm}$ ) and quantification (right, $n=3$ for each group). Data represent means \pm s.d. $t$-test. ${ }^{\star \star \star} P<0.001$ (e) The relative mRNA levels of Med23, Runx2, Osx, Alp and Ocn were quantified by RT-PCR. Data represent means \pm s.d. All data represent means \pm s.d. $t$-test, ${ }^{\star \star} P<0.01,{ }^{\star \star \star} P<0.001$.

were significantly reduced in $\mathrm{Med} 23_{\mathrm{MSC}}{ }^{-/-}$mice compared with controls, while Runx2 and Atf4, another key factor involved in osteogenesis, were unchanged (Fig. 4e). Consistently, Von Kossa staining and immunohistochemical analyses of bone tissue sections from E16.5 control and $\mathrm{Med} 23_{\mathrm{MSC}^{-/}}$embryos further confirmed the defects in bone formation and impaired osteoblast differentiation in vivo (Fig. 4f,g). Similarly, defects in ossification and decrease in osteogenic gene expression were observed in $\mathrm{Med} 23_{\mathrm{ob}}-1-$ mice (Supplementary Fig. 3c-e). Both the in vitro and in vivo experiments thus showed that Med23 deletion reduced the expression of multiple Runx2-target genes while not affecting the expression of Runx2 itself. These observations implied that MED23 may participate in osteoblast differentiation and bone development by controlling RUNX2 activity.

Med23 modulates Runx 2 activity as a cofactor. We next set out to investigate whether MED23 controls the transcriptional activity of Runx2. To this end, we infected murine C3H10T1/2 embryonic fibroblasts with retroviruses expressing control or Med23 knockdown shRNAs and then transfected the stable cells with a luciferase reporter driven by the Ocn promoter (OC1050-Luc), which is a prototypical Runx2-target gene promoter that is widely used as a tool to study the molecular regulation of osteoblast development ${ }^{24}$. In the absence of MED23,
RUNX2-dependent activation of the Ocn promoter was significantly inhibited (Fig. 5a). The Ocn promoter harbours two binding sites: OSE2 and OSE1, which are specifically bound by Runx2 and ATF4, respectively ${ }^{25}$. To discriminate the effects of Med23 deficiency on the two key factors, we performed the luciferase reporter assay with either 6XOSE2-Luc or 6XOSE1-Luc in control and Med23 shRNA knockdown cells. Med23 deficiency specifically impaired the Runx2driven activation of 6XOSE2-Luc but did not affect the ATF4-driven activation of 6XOSE1-Luc, suggesting a specific connection between Med23 and Runx2 (Fig. 5b,c). To further verify the specific regulation of RUNX2 activity by MED23, we performed a titration experiment with low and high doses of MED23 co-transfected with the OC1050Luc reporter, which showed that RUNX2 activity was repressed gradually with increasing dose of MED23 (Fig. 5d). The repressive effect on RUNX2 activity by overexpression of MED23 was also confirmed by 6XOSE2-Luc reporter system (Supplementary Fig. 7a). By contrast, overexpression of MED23 did not affect ATF4 activity (Fig. 5d and Supplementary Fig. 7b). RUNX2 activity was quenched by high level overexpression of MED23 probably in that the exogenous MED23 may compete with endogenous MED23 within the Mediator complex to prevent the transcription factor Runx2 from recruiting the Mediator complex.

Cooperative activation of the Ocn promoter by MED23 and RUNX2 led us to examine the physical interaction between the two molecules. We transfected 293 T cells with tagged Runx 2 and Med23 and performed a co-immunoprecipitation (co-IP) assay, 
a

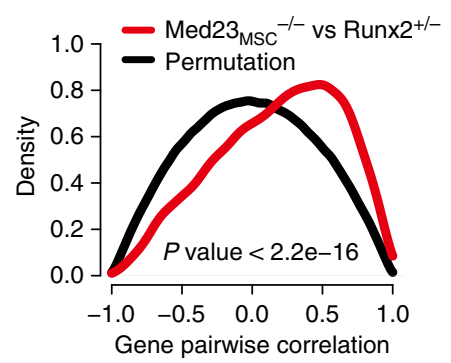

C

d
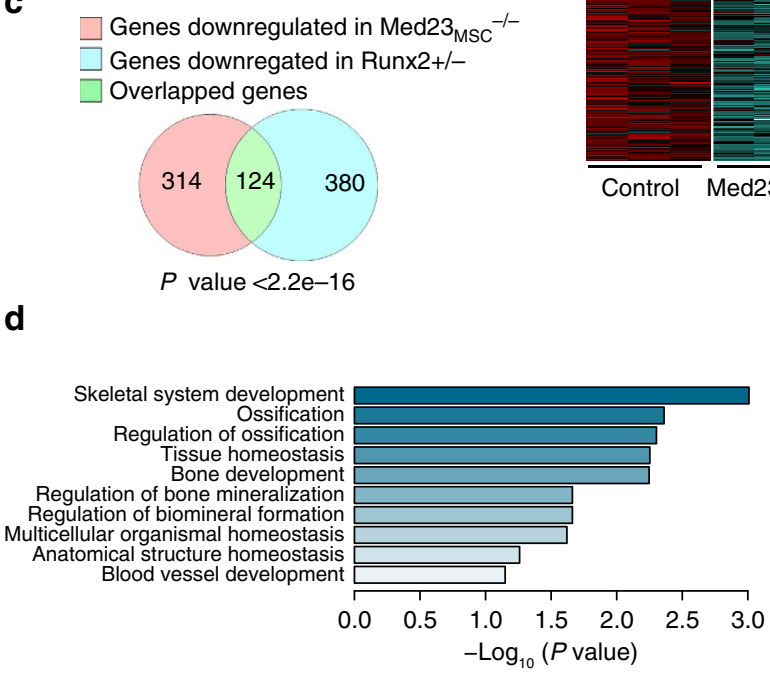

b

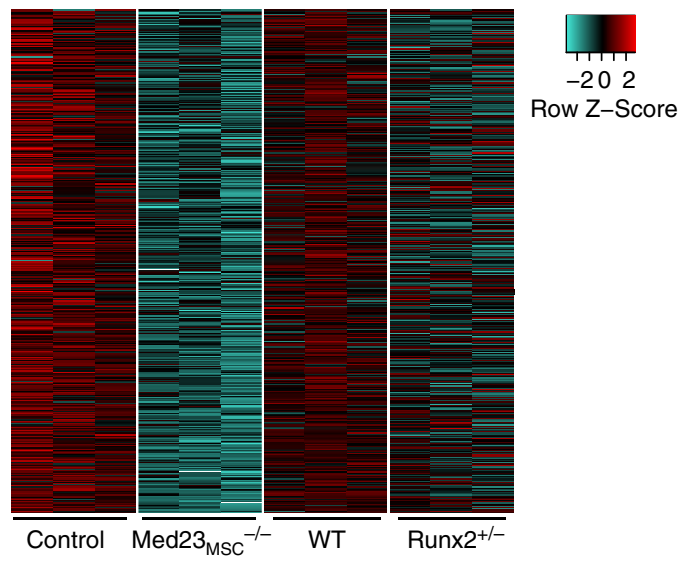

f

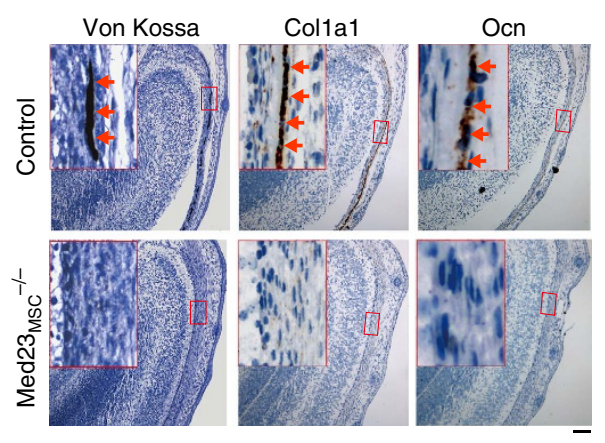

g

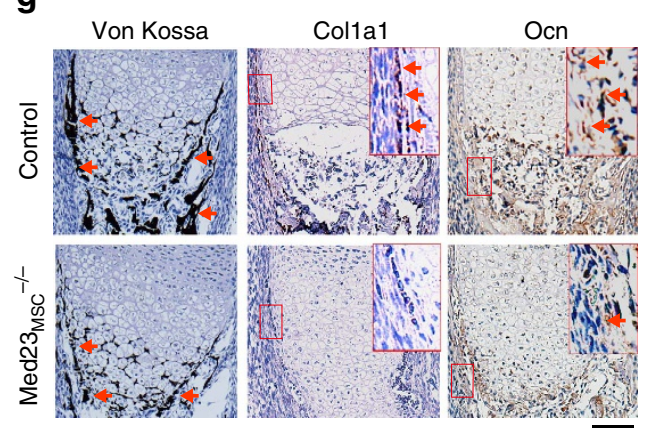

Figure 4 | Med23 regulates expression of genes for osteoblast development. (a) Pair-wise correlation analysis of global gene expression in control versus

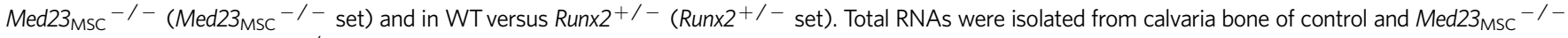
newborns and WT and Runx $2^{+/-}$newborns, followed by RNA sequencing analysis. Kolmogorov-Smirnov (K-S) test was used for testing for correlation between

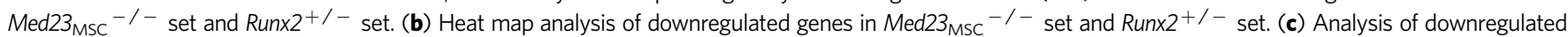
genes ( $\log _{2}$ fold change $>0.5$ ) common to $M e d 23_{\mathrm{MsC}}-/$ - set and Runx $2^{+/-}$set. (d) Gene ontology (GO) enrichment analysis of downregulated genes ( $>1.5$-fold) in Med23 $\mathrm{MSC}^{-1-}$ set. (e) Relative expression level of osteogenic marker mRNAs from parietal bones of P2 control and Med23 $\mathrm{MSC}^{-/-}$. Data represent means \pm s.d. $t$-test, ${ }^{\star} P<0.05$ and ${ }^{\star \star \star} P P<0.001$. (f) Sections $(4 \mu \mathrm{m})$ of skull tissues and $(\mathbf{g})$ sections $(4 \mu \mathrm{m})$ of tibia bone from E16.5 control and Med23 $\mathrm{MSC}^{-/-}$embryos were analysed for bone mineralization by von Kossa staining (left), and for expression of Colla1 (middle) and Osteocalcin (Ocn) (right) by in situ hybridization. Scale bar, $100 \mu \mathrm{m}$.

which revealed that MED23 physically associated with RUNX2 (Fig. 5e). Consistent with these results, endogenous RUNX2 and MED23 interacted in differentiated MC3T3E1 osteoblastic cells (Fig. 5f). The interaction with MED23 was mediated via the Runt and PST domains of RUNX2 (Supplementary Fig. 8a,b). GST pull-down assay implied that MED23/RUNX2 interaction was likely direct (Supplementary Fig. 8c). Moreover, immunofluorescence staining showed that these two proteins largely colocalized in the nucleus (Fig. 5g). As gene transcription involves a key step in which the activators work with the Mediator complex to recruit RNA Pol II to the gene promoters, we next used a chromatin IP
(ChIP) assay to test whether Med23 deletion affected the recruitment of RNA Pol II by RUNX2 and the binding of RUNX2 to its target's promoter. The result showed that the occupancy of Pol II at the promoter of Ocn, a direct target gene of Runx2, was markedly decreased in the absence of MED23 while RUNX2 occupancy did not alter, suggesting that Med23 deficiency impaired the recruitment of Pol II to the promoter of Runx2-target osteogenic marker genes (Fig. 5h). Taken together, these results suggest that the Mediator subunit MED23 acts as a coactivator of Runx2 to regulate osteoblast differentiation. 
a
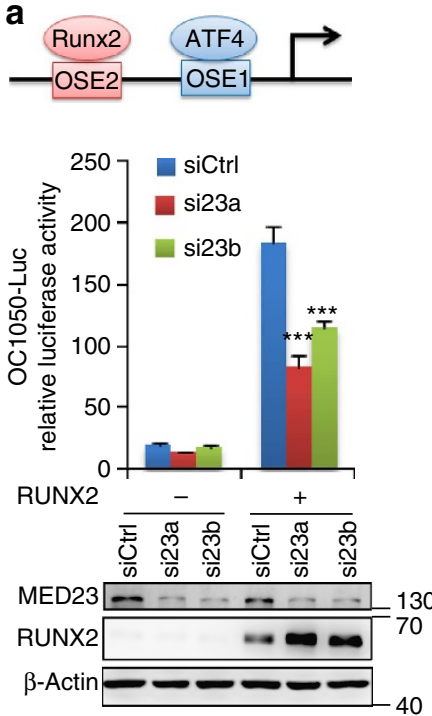

d

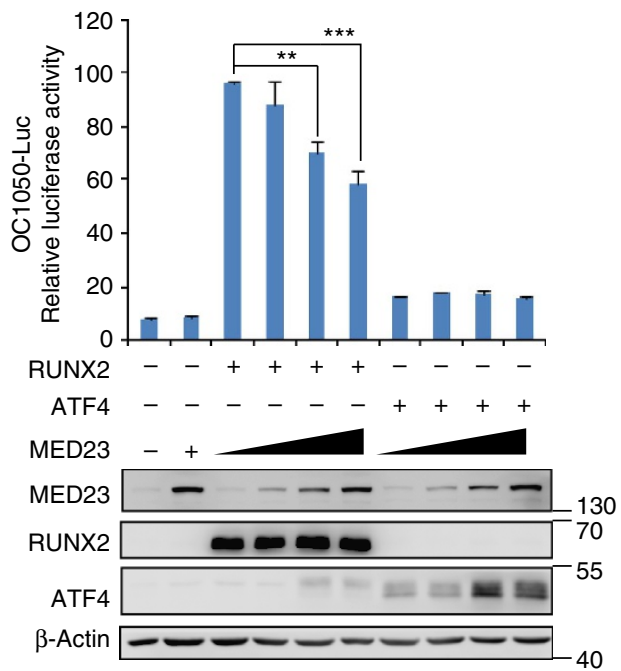

$\mathbf{h}$

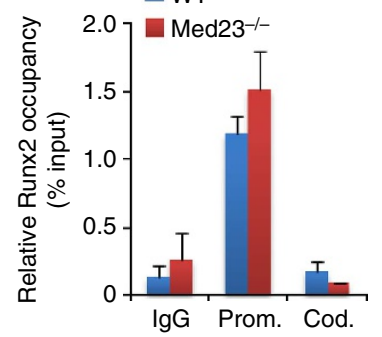

b
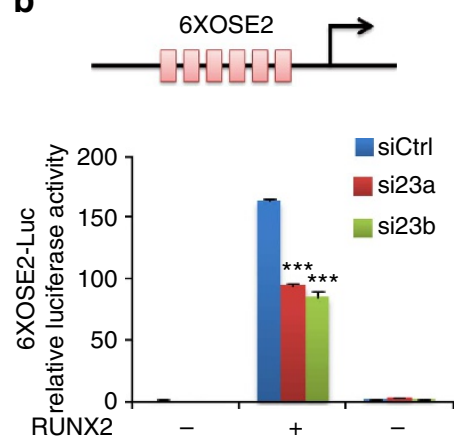

ATF4

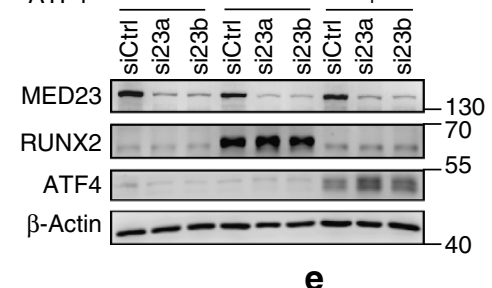

C
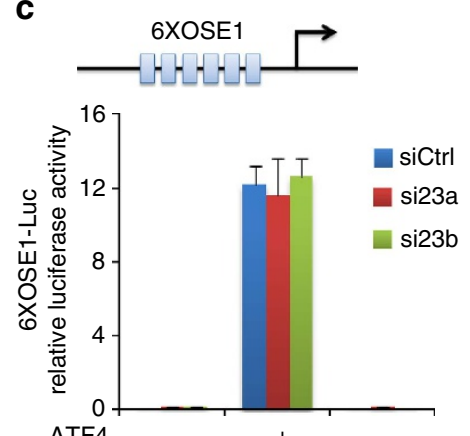

RUNX2
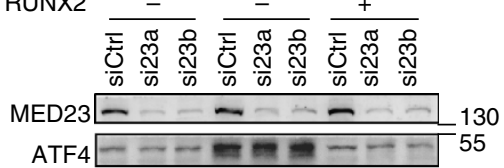

RUNX2 $\square-70$

$\beta$-Actin $-\infty-\infty-\infty$ 40
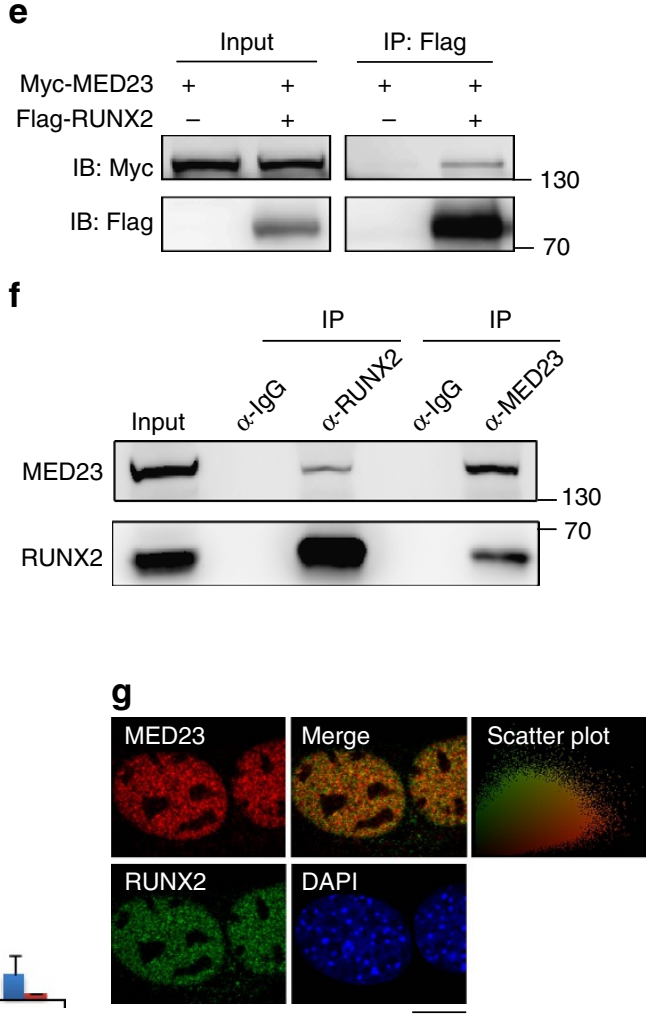

Figure 5 | Regulation of RUNX2 transcriptional activity by MED23. (a-c) Effect of Med23 deficiency on Runx2-mediated activation of the osteocalcin tested with luciferase expression report systems (a) OC1050-Luc, (b) 6XOSE2-Luc and (c) 6XOSE1-Luc. Luciferase assay was performed in control and Med23 knockdown C310T1/2 cells. $n=3$ for each group, all data represent means \pm s.d. $t$-test, ${ }^{\star \star \star} P<0.001$ (d) Effects of an increasing amount of MED23 on transcriptional activity of Runx2 in C310T1/2 cells with the OC1050-Luc report system. Below is the western blot analysis of MED23 levels in the lysates. $n=3$ for each group, all data represent means \pm s.d. $t$-test. ${ }^{\star \star} P<0.01,{ }^{\star \star \star} P<0.001$ (e) Co-immunoprecipitation (Co-IP) of MED23 with Flag-RUNX2. Flag-RUNX2 expressing plasmid was co-transfected with Myc-Med23 into 293T cells. Whole cell lysate was used for immunoprecipitation and then immunoblotting with indicated antibodies. (f) Physical interaction between endogenous RUNX2 and MED23. Co-IP experiment was performed in BMP2-stimulated MC3T3E1 cells. Whole cell lysate was used for immunoprecipitation with anti-RUNX2 or anti-MED23, followed by detection with indicated antibodies by western blot. (g) Co-localization assay in primary osteoblast cells. Cells were IF stained with antibodies against MED23 and RUNX2 after stimulated with BMP2 for $24 \mathrm{~h}$. Scale bar, $10 \mu \mathrm{m}$. The scatter plot showed FITC and RRX emission intensities were plotted on $x$ - and $y$-axes, respectively. Co-localization was analysed by the Volocity software, which showed the coefficient for co-localization of $0.786 \pm 0.003$ (Pearson's correlation coefficient). (h,i) ChIP assay for occupation of RUNX2 (h) or Pol II (i) on the promoter of Osteocalcin. Immortalized WT and Med23-/ - bone marrow-derived mesenchymal cells were cultured in osteogenic medium for 6 days, followed by fixation and lysation. Chromatin from cell lysates was immunoprecipited with anti-RUNX2 or anti-Pol II and quantified by RT-PCR. 'Prom' represents the promoter region and 'Cod' represents the coding region. $n=3$ for each group, all data represent means \pm s.d. $t$-test, ${ }^{\star \star} P<0.01$. 
a

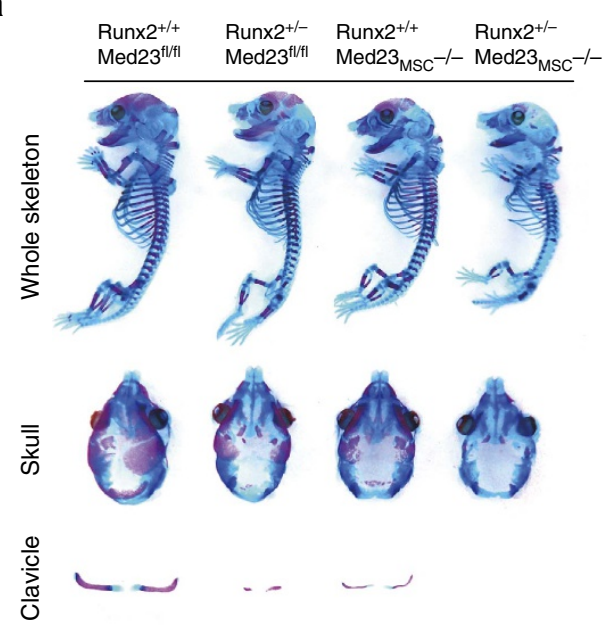

b

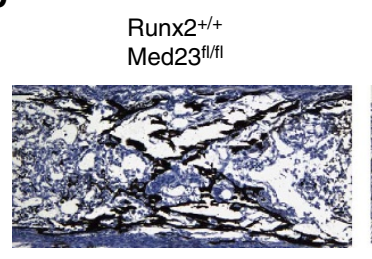

C

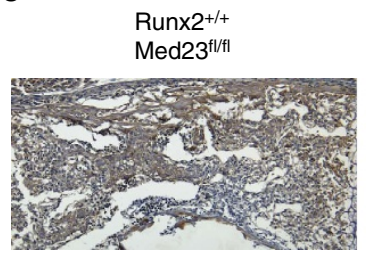

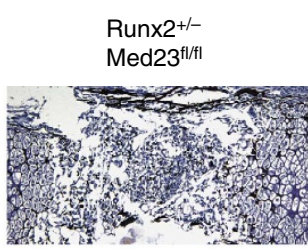

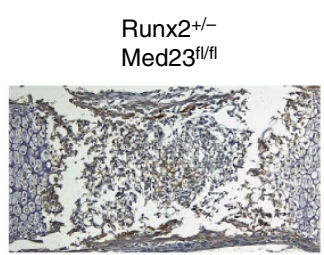

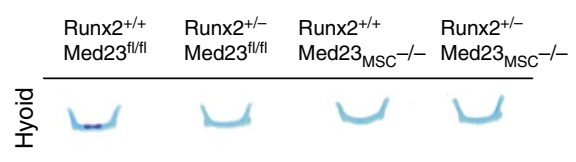
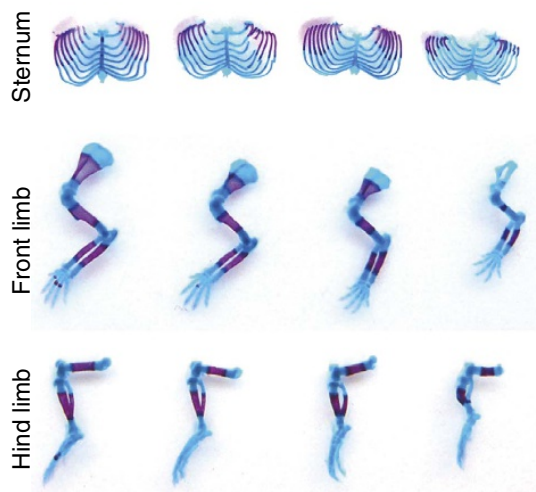

Runx2+/Med23 $\mathrm{MSC}^{-1}$
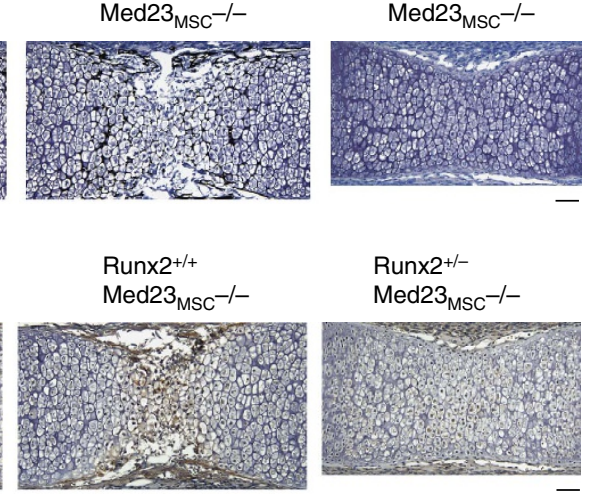

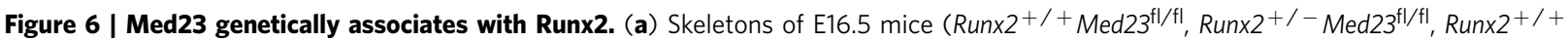
Med23 $\mathrm{Msc}^{-/-}$, Runx2 ${ }^{+/-}$Med23 $\mathrm{Msc}^{-/-}$) were double stained by alizarin red/alcian blue. (b) Von Kossa staining of humeri from E15.5 mice

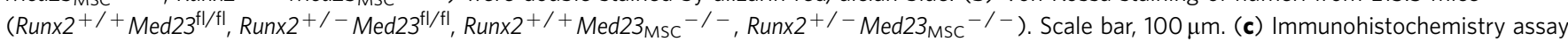

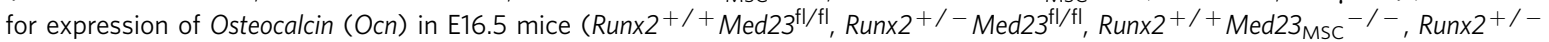

Med $23_{\mathrm{MSC}^{-/-}}$). Scale bar, $100 \mu \mathrm{m}$.

Med23 genetically interacts with Runx2 during osteogenesis. Based on the in vitro findings described above, we next questioned whether MED23 synergized with RUNX2 in vivo. We hypothesized that if MED23 acts together with RUNX2 to regulate osteoblast activity, then deletion of $\mathrm{Med} 23$ in parallel with Runx2 haploinsufficiency in vivo should aggravate the skeletal defects observed in Runx2 $2^{+/-}$mice. To test this hypothesis and since disruption in single copy of $\mathrm{Med} 23 \mathrm{did}$ not reduce the protein level of MED23, we crossed Med23 $\mathrm{MSC}^{-1-}$ mice with Med $23^{\mathrm{fl} / \mathrm{fl}} / \mathrm{Run}_{\mathrm{x}} 2^{+/-}$mice and analysed the skeletal phenotypes of E16.5 embryos by alcian blue/ARS double staining. As shown in Fig. 6a, both $\mathrm{Med} 23^{\mathrm{fl} / \mathrm{fl}} / \mathrm{Runx} 2^{+I-}$ mice and Med $23_{\mathrm{MSC}^{-}}{ }^{-}$ mice showed the previously reported CCD-like skeletal abnormalities observed in heterozygous Runx2 mutant mice ${ }^{8,10}$. Run $\times 2^{+1-} /$ Med $23_{\mathrm{MSC}^{-1}}$ mice displayed significant bone defects, including delayed ossification of the fontanelles and hypoplasia of the clavicles (Fig. 6a). Furthermore, Von Kossa staining revealed reduced ossification of the long bones of E16.5 Runx $2^{+1-}$ and $\mathrm{Med} 23_{\mathrm{MSC}}{ }^{-1-}$ mice compared with control mice. However, Run $\times 2^{+1-} / \mathrm{Med} 23_{\mathrm{MSC}}{ }^{-1-}$ compound mutant mice displayed a more severe reduction in ossification (Fig. 6b). Consistent with the reduced Von Kossa staining, the expression of Ocn was decreased significantly in the Runx $2^{+/-} / \mathrm{Med} 23_{\mathrm{MSC}^{-1}}$ compound mutant mice compared with the Runx2 $2^{+1-}$ mice and $M e d 23_{\mathrm{MSC}}{ }^{-1-}$ mice
(Fig. 6c). Taken together, these data indicate a genetic interaction between Med23 and Runx2 and provide in vivo verification that MED23 can regulate osteoblast function via cooperation with RUNX2.

\section{Discussion}

The present study provides several lines of evidence that Med23 is required for osteoblast differentiation and bone development. Homozygous loss of Med23 in mesenchymal progenitors resulted in prominent defects in bone development, including hypoplasia of the clavicles, retarded ossification of the cranial bones and reductions in the trabecular bone and overall bone mass. Furthermore, Med23 conditional knockout in preosteoblasts largely recapitulated the characteristics of $\mathrm{Med} 23_{\mathrm{MSC}}{ }^{-1-}$ mice. Phenotypic analysis showed that Med23 deficiency affected both intramembranous and endochondral bone formation. In vitro differentiation experiments indicated that the defective osteogenesis happened in a cell-autonomous manner. In contrast to the impaired osteoblast development, the presence of multinucleated osteoclasts in the mineralized cartilage matrix of Med23-deficient mice indicated that osteoclast differentiation occurred normally.

MSCs can differentiate into different lineages, including osteoblasts, adipocytes, chondrocytes and myocytes ${ }^{1,2}$. Different 
transcription factors have been shown to specify distinct cell fates. In addition to the function of Runx2 in osteoblast differentiation, PPAR $\gamma$, Sox 9 and $M y o D$ drive MSCs to differentiate into adipocytes, chondrocytes and myocytes, respectively $7,8,26-28$. The role of the Mediator complex in PPAR $\gamma$-mediated adipogenesis has been established by observing a direct interaction between MED1 and PPAR $\gamma^{13}$. For Sox9, studies from the zebra fish model indicated that Trap230/MED12 can function as a coactivator for SOX9 during cartilage development, with Trap230/Med12 mutant zebra fish strikingly resembling the Sox9 mutant phenotype ${ }^{29}$. Our previous study demonstrated that MED23 represses smooth muscle cell differentiation while facilitating adipocyte differentiation in multipotent MSCs ${ }^{16}$, indicating that a single Mediator complex subunit is able to regulate the differentiation of MSCs into various cell lineages, either positively or negatively, by cooperating with lineagespecifying transcription factors such as ELK1 and MAL.

To date, there have been no reports of a direct role for the Mediator complex in RUNX2 function. Our study demonstrated that MED23 can act together with RUNX2 to drive osteoblast differentiation. To the best of our knowledge, this is the first report of a direct role for the Mediator complex in modulating RUNX2 function. We provide evidence that the Mediator complex subunit MED23 partially regulates the transcriptional activity of RUNX2 and is required for osteoblast differentiation and bone development. First, homozygous loss of Med23 in mesenchymal progenitors resembled the bone phenotype of Runx2 heterozygous mice, resulting in prominent defects in bone development, with hypoplasia of the clavicles, retarded ossification of the cranial bones and reduction in the trabecular bone and overall bone mass. Moreover, Med23 conditional knockout in preosteoblasts largely recapitulated the characteristics of Med $23_{\mathrm{MSC}^{-1}}$ mice, with abnormalities in multiple skeletal elements. Interestingly, Med23 deficiency did not alter RUNX2 expression but instead caused the downregulation of multiple Runx2-regulated genes, such as Osx and Ocn. Reporter assays revealed that $M e d 23$ deficiency impaired Runx2 transcriptional activity but did not affect the activity of ATF4, another key regulator of osteoblast differentiation. Biochemical analyses further established the physical association between RUNX2 and MED23. Finally, Med23 deletion further aggravated the defective skeletal phenotype of $R u n \times 2^{+1-}$ mice. In addition to the function in osteoblasts, Runx2 is also found to control the maturation of chondrocytes through Runx2 conditional knockout in chondrocytes by Col2al-Cre $e^{30,31}$. Histological analysis of tibia showed that chondrocytes were arrested during terminal maturation in $\mathrm{Med} 23_{\mathrm{MSC}}{ }^{-/-}$mice compared with control littermates as evidenced by less endochondral ossification and smaller hypertrophic chondrocytes in $\mathrm{Med} 23_{\mathrm{MSC}}{ }^{-1-}$ mice (Supplementary Fig. 10a-c). All the results described above provide in vivo genetic evidence that MED23 is a cofactor of RUNX2.

Runx2 mutations are known to underlie human CCD, an autosomal-dominant heritable skeletal disease that is typically characterized by open or delayed closure of calvarial fontanelles and clavicle hypoplasia $9,32,33$. This phenotype can be reproduced in heterozygous Runx2 mutant mice ${ }^{10}$. More precisely, a $70 \%$ reduction in Runx2 levels also generates the CCD phenotype in mice ${ }^{34}$. Indeed, human bone marrow-derived MSCs with Med23 deficiency were retarded to differentiate into osteoblasts, which might imply that MED23 plays a role in human skeletal development and its mutation may relate to human bone diseases (Supplementary Fig. 9). In addition, the dysregulation of Runx2 activity through manipulation of numerous nuclear factors, for example, TAZ, MAF and Satb2, causes abnormal osteoblast differentiation and bone development ${ }^{11,35,36}$. These studies indicate that the Runx2-dependent transcriptional output is under fine surveillance via the cooperation of many factors. Our study demonstrated that the Mediator subunit MED23 acts as a cofactor of RUNX2 in the regulation of osteoblast differentiation and bone development, providing an insight into the regulation of RUNX2 activity and skeletal dysplasias such as CCD.

\section{Methods}

Mice. Med23-floxed mice were generated by homologous recombination ${ }^{16,17}$ Briefly, the exons 5-7 were flanked by two loxP sites. After targeting vector delivery, ES cells were screened by PCR. Med23-floxed mice were backcrossed with C57/BL6J mice for at least six generations. Prx1-Cre and Osx-Cre-ER mice were purchased from the Jackson Laboratory. Run $\times 2^{+/-}$mice were kindly provided by Professor Laurie Glimcher's lab. All mice were maintained under specific pathogen-free conditions. All animal experimental procedures were approved by the Institutional Animal Care and Research Advisory Committee of the Shanghai Institute of Biochemistry and Cell Biology.

Analysis of bone phenotypes. Skeletal preparations were double stained with alcian blue and $\mathrm{ARS}^{8,10}$. Briefly, embryos or newborns were eviscerated and the skin was removed. After fixation with $95 \%$ ethanol for 3 days, embryos or newborns were stained for 3 days in Alcian blue solution. Then they were fixed and cleared with $95 \%$ ethanol for three times and each $1.5 \mathrm{~h}$, followed by treatment of $2 \% \mathrm{KOH}$ for 3-4h. After stained with ARS solution for 3-4h, skeletons were cleared in $1 \% \mathrm{KOH} / 20 \%$ glycerol. For histological analysis, bone tissues were fixed in $4 \%$ paraformaldehyde (PFA) and then embedded in paraffin. For embryonic mice, $4 \mu \mathrm{m}$ tissue sections were used for Von Kossa staining, DIG labelled in situ hybridization (Roche) and immunohistochemical staining (Dako). For postnatal mice, bone tissues were fixed in $4 \%$ PFA and decalcified for 2 weeks prior to paraffin embedding. Tissue sections $(4 \mu \mathrm{m})$ were used for TRAP staining according to the standard protocol.

Measurement of PINP concentrations. We determined serum concentrations of PINP using the mouse PINP EIA kit (Immunodiagnostic Systems) according to the instructions provided.

Micro CT analysis. Mouse hind limbs were harvested, soft tissues were removed and the remaining tissues were stored in $70 \%$ ethanol. Scanning was performed with a Skyscan 1076 instrument, and 36 slides ( $18 \mu \mathrm{m}$ each) immediately below the growth plate in the distal metaphysis of the femur were used for quantification of the bone parameters.

Cell culture. C310T1/2 (ATCC) and 293T (ATCC) cells were maintained in DMEM containing 10\% FBS. MC3T3E1 (ATCC) cells were maintained in Minimum Essential Medium $\alpha$ ( $\alpha$-MEM) containing 10\% FBS. MSCs were isolated from bone marrow samples of 6 - to 8 -week-old mice and cultured in $\alpha$-MEM containing $15 \%$ FBS. All cells were cultured in a $5 \% \mathrm{CO}_{2}$ humidified incubator at $37^{\circ} \mathrm{C}$. For the c.f.u.-F assay, $2 \times 10^{6}$ bone marrow-derived MSCs or $1 \times 10^{2}$ osteoblast cells from calvaria were plated on each well of a six-well plate, cultured for 2 weeks and then fixed in $4 \%$ PFA and stained with $0.5 \%$ crystal violet, followed by counting the stained colonies. For in vitro osteoblast differentiation, MSCs were cultured in osteogenic medium (10\% FBS with $50 \mathrm{\mu g} \mathrm{ml}^{-1}$ ascorbic acid, $10 \mathrm{nM}$ dexamethasone and $10 \mathrm{mM} \beta$-glycerophosphate) and subjected to ALP staining on day 7 and ARS staining on day 21. Human bone marrow-derived MSCs were cultured and induced to differentiate into osteoblasts according to the protocol from ScienCell.

Transient transfection and luciferase reporter assay. C3H10T1/2 cells were seeded overnight at $4 \times 10^{4}$ cells per well into a 24 -well plate and transfected by Lipofectamine 2000 (Life Technologies) with a luciferase reporter plasmid and pRL-TK (Promega) along with various expression constructs, as indicated. All wells were supplemented with control empty expression vector plasmids to keep the total amount of DNA constant. At $36-48 \mathrm{~h}$ post transfection, the cells were harvested and subjected to dual-luciferase reporter assays according to the manufacturer's protocol (Promega).

IP and immunoblotting. 293T cells were seeded at $6 \times 10^{6}$ cells per $10 \mathrm{~cm}$ dish and cultured overnight. At $36-48 \mathrm{~h}$ after transfection with Lipofectamine 2000, cells were harvested and lysed in lysis buffer ( $1 \% \mathrm{NP}-40,10 \%$ glycerol, $135 \mathrm{mM} \mathrm{NaCl}$ $20 \mathrm{mM}$ Tris, $\mathrm{pH}$ 8.0) supplemented with protease inhibitors. Lysates were subjected to IP with anti-Flag antibodies (M2, Sigma) at $4{ }^{\circ} \mathrm{C}$ overnight, followed by washing in lysis buffer, SDS-PAGE electrophoresis and immunoblotting with the indicated antibody (1:2,000, anti-Myc, Sigma). To investigate endogenous protein-protein interactions, MC3T3E1 cells were cultured for 4 days in $\alpha$-MEM with $10 \%$ FBS 
supplemented with $100 \mathrm{ng} \mathrm{ml}^{-1} \mathrm{BMP} 2,50 \mu \mathrm{g} \mathrm{ml}^{-1}$ ascorbic acid and $10 \mathrm{mM}$ $\beta$-glycerophosphate. Two hours prior to harvest, MG132 (10 $\mu \mathrm{M}$, Sigma) was added to all cultures. Harvested cells were subjected to lysis and IP with antiRUNX2 (Sigma), anti-MED23 (Novus) or control IgG, followed by washing in lysis buffer, SDS-PAGE electrophoresis and immunoblotting with the indicated antibody (Anti-MED23, 1:1,000, BD Biosciences; anti-Runx2, 1:1,000, MBL). Primary bone marrow MSCs or bone tissues were lysed in RIPA buffer ( $50 \mathrm{mM}$ Tris pH7.4, $150 \mathrm{mM} \mathrm{NaCl}, 1 \%$ Triton X-100, $1 \%$ sodium deoxycholate, $0.1 \%$ SDS) in presence of protease inhibitors. Whole cell lysate were centrifuged and then subjected to western blots according to standard protocols. Uncropped results for all western blots are shown in Supplementary Fig. 11.

In vitro interaction assay. GST or GST-RUNX2 recombined proteins were expressed in Escherichia coli BL21, followed by purification according to the instructions of the manufacturer (GE). His-Flag-MED23 was expressed using the Bac-to-Bac baculovirus expression system and purified by Ni-NTA agarose beads (Invitrogen). Purified GST or GST-Runx2 was incubated with purified MED23 and subjected to GST pull-down experiment . The bound proteins were analysed by SDS-10\% PAGE and immunoblotting with indicated antibodies (Anti-GST, 1:1,000, Santa Cruz; anti-MED23, 1:1,000)

Immunofluorescence staining and co-localization analysis. Primary osteoblast cells were plated on cover slips for $24 \mathrm{~h}$ in $\alpha$-MEM with $10 \%$ FBS with BMP2 $\left(100 \mathrm{ng} \mathrm{ml}^{-1}\right)$ prior to staining. After removal of culture media, cells were washed with PBS, fixed in $4 \%$ PFA, permeabilized with $0.2 \%$ Triton $\mathrm{X}-100$ for $10 \mathrm{~min}$ and then blocked with 2\% BSA in PBS for $1 \mathrm{~h}$. Anti-MED23 (1:100) and anti-RUNX2 (1:200) primary antibodies were diluted in the blocking solution and applied overnight at $4^{\circ} \mathrm{C}$. After PBS wash for three times, secondary antibodies (Jackson Laboratory) were diluted (1:100) in blocking buffer and applied for $1 \mathrm{~h}$ at room temperature. After PBS wash for three times, nuclei were stained with DAPI $(1: 4,000)$ for $4 \mathrm{~min}$. Co-localization analysis were performed using Volocity Software (PerkinElmer)

Real-time PCR analysis. Total RNA was isolated from bone tissue or cells with TRIzol reagent (Life Technologies). Complementary DNA was generated using M-MLV reverse transcription kit (Promega). Real-time PCR was conducted in triplicate with SYBR Premix Ex Taq (Takara). The level of the endogenous mRNA was normalized to the level of Gapdh mRNA using the $2^{-\Delta \mathrm{CT}}$ method. Specific primer sequences are listed in the Supplementary Table 1.

ChIP assay. Cells were cross-linked with $1 \%$ formaldehyde in culture media for $10 \mathrm{~min}$ at room temperature, followed by adding $0.125 \mathrm{M}$ glycine to quench the cross-linking. Cells were washed and scraped in cold PBS and harvested in ChIP lysis buffer (50 mM Tris-HCl, pH 7.4, 1\% SDS, 10 mM EDTA). Cell lysates were then sonicated to shear chromatin. After that, cell lysates were centrifuged and $100 \mu \mathrm{l}$ supernatant was diluted with $900 \mu \mathrm{l}$ dilution buffer $(16.7 \mathrm{mM}$ Tris- $\mathrm{HCl}$, pH 8.0, $0.01 \%$ SDS, $1.1 \%$ Triton X-100, $1.2 \mathrm{mM}$ EDTA, $167 \mathrm{mM} \mathrm{NaCl}$ ) and then incubated with $2 \mu \mathrm{g}$ anti-RUNX2 or $2 \mu \mathrm{g}$ anti-Pol II (Santa Cruz) or non-immune Rabbit IgG overnight at $4^{\circ} \mathrm{C}$. About $20 \mu \mathrm{l}$ Protein $\mathrm{G}$ magnetic beads (Life technologies) were added for another $4 \mathrm{~h}$ on a rotating wheel. Chromatin then was immunoprecipitated, decrosslinked at $65^{\circ} \mathrm{C}$ and treated with Proteinase K. Precipitated DNA was extracted and quantified by real-time PCR. All values were normalized to input. Specific primer sequences were listed in Supplementary Table 1 .

Retrovirus infection. Retroviruses expressing RNA interference (RNAi) oligonucleotides were generated by transfection of recombinant pSiren-RetroQ and pCL10A1 helper plasmids into 293T cells using Lipofectamine 2000. Culture media were changed $24 \mathrm{~h}$ after transfection and the supernatants containing retroviruses were harvested $48 \mathrm{~h}$ later and passed through a $0.45-\mu \mathrm{m}$ filter. Filtered supernatants were supplemented with $6 \mu \mathrm{g} \mathrm{ml}^{-1}$ polybrene and added to the cells grown in sixwell plates for centrifugation at 2,500 r.p.m. for $1.5 \mathrm{~h}$ at $30^{\circ} \mathrm{C}$. About $24 \mathrm{~h}$ after spin infection, infected cells were then selected in the presence of puromycin: primary MSCs at $5 \mu \mathrm{g} \mathrm{ml}^{-1}$ and C3H10T1/2 at $8 \mu \mathrm{g} \mathrm{ml}^{-1}$. The RNAi oligo sequences are listed in Supplementary Table 2.

RNA-Seq and GO analysis. Total RNA was isolated with TRIzol from the parietal bone of P2 control mice $(n=3)$ and $\mathrm{Med} 23_{\mathrm{MSC}}{ }^{-1-}$ littermates $(n=3)$ and from the parietal bone of P2 WT mice $(n=3)$ and Runx2 $2^{+1-}$ mice $(n=3)$. Complementary DNA library preparation and sequencing were performed according to the Illumina standard protocol. GO analysis was performed with the DAVID online tool. Top GO categories were selected according to the $P$ values. RNA-seq data in this study have been deposited at Gene Expression Omnibus (GEO) (http:// www.ncbi.nlm.nih.gov/gen/) under accession ID GSE 77007.
Statistical analysis. Statistical analyses were performed with two-tailed, unpaired Student's $t$-test. Kolmogorov-Smirnov test was used to test for comparing cumulative distributions of two data sets (Fig. 4a).

\section{References}

1. Harada, S.-I. \& Rodan, G. A. Control of osteoblast function and regulation of bone mass. Nature 423, 349-355 (2003).

2. Mendez-Ferrer, S. et al. Mesenchymal and haematopoietic stem cells form a unique bone marrow niche. Nature 466, 829-834 (2010).

3. Maxson, S., Lopez, E. A., Yoo, D., Danilkovitch-Miagkova, A. \& LeRoux, M. A Concise review: role of mesenchymal stem cells in wound repair. Stem Cells Transl. Med. 1, 2011-0018 (2012).

4. Parekkadan, B. \& Milwid, J. M. Mesenchymal stem cells as therapeutics. Annu. Rev. Biomed. Eng. 12, 87-117 (2010).

5. Kitada, M. \& Dezawa, M. Parkinson's disease and mesenchymal stem cells: potential for cell-based therapy. Parkinsons Dis. 2012, 873706-873714 (2012)

6. Au, P., Tam, J., Fukumura, D. \& Jain, R. K. Bone marrow derived mesenchymal stem cells facilitate engineering of long-lasting functional vasculature. Blood 111, 4551-4558 (2008).

7. Ducy, P., Zhang, R., Geoffroy, V., Ridall, A. L. \& Karsenty, G. Osf2/Cbfa1: a transcriptional activator of osteoblast differentiation. Cell 89, 747-754 (1997).

8. Komori, T. et al. Targeted disruption of Cbfal results in a complete lack of bone formation owing to maturational arrest of osteoblasts. Cell 89, 755-764 (1997).

9. Mundlos, S. et al. Mutations involving the transcription factor CBFA1 cause cleidocranial dysplasia. Cell 89, 773-779 (1997).

10. Otto, F. et al. Cbfa1, a candidate gene for cleidocranial dysplasia syndrome, is essential for osteoblast differentiation and bone development. Cell 89, 765-771 (1997).

11. Hong, J.-H. et al. TAZ, a transcriptional modulator of mesenchymal stem cell differentiation. Science 309, 1074-1078 (2005).

12. Hanai, J.-I. et al. Interaction and functional cooperation of PEBP2/CBF with Smads. J. Biol. Chem. 274, 31577-31582 (1999).

13. Ge, K. et al. Transcription coactivator TRAP220 is required for PPAR[gamma]2-stimulated adipogenesis. Nature 417, 563-567 (2002).

14. Yin, J.-W. \& Wang, G. The Mediator complex: a master coordinator of transcription and cell lineage development. Development 141, 977-987 (2014).

15. Wang, W. et al. Mediator MED23 links insulin signaling to the adipogenesis transcription cascade. Dev. Cell 16, 764-771 (2009).

16. Yin, J.-W. et al. Mediator MED23 plays opposing roles in directing smooth muscle cell and adipocyte differentiation. Genes Dev. 26, 2192-2205 (2012).

17. Chu, Y. et al. Liver Med23 ablation improves glucose and lipid metabolism through modulating FOXO1 activity. Cell Res. 24, 1250-1265 (2014).

18. Logan, M. et al. Expression of Cre recombinase in the developing mouse limb bud driven by a Prxl enhancer. Genesis 33, 77-80 (2002).

19. Woo, W.-M., Zhen, H. H. \& Oro, A. E. Shh maintains dermal papilla identity and hair morphogenesis via a Noggin-Shh regulatory loop. Genes Dev. 26, 1235-1246 (2012).

20. Cao, X. Targeting osteoclast-osteoblast communication. Nat. Med. 17, 1344-1346 (2011)

21. Rodda, S. J. \& McMahon, A. P. Distinct roles for Hedgehog and canonical Wnt signaling in specification, differentiation and maintenance of osteoblast progenitors. Development 133, 3231-3244 (2006).

22. Davey, R. et al. Decreased body weight in young Osterix-Cre transgenic mice results in delayed cortical bone expansion and accrual. Transgenic Res. 21 , 885-893 (2012).

23. Huang, W. \& Olsen, B. Skeletal defects in Osterix-Cre transgenic mice. Transgenic Res. 24, 167-172 (2015).

24. Ducy, P. \& Karsenty, G. Two distinct osteoblast-specific cis-acting elements control expression of a mouse osteocalcin gene. Mol. Cell. Biol. 15, 1858-1869 (1995).

25. Yang, X. et al. ATF4 Is a substrate of RSK2 and an essential regulator of osteoblast biology: implication for Coffin-Lowry syndrome. Cell 117, 387-398 (2004).

26. Tontonoz, P., Hu, E. \& Spiegelman, B. M. Stimulation of adipogenesis in fibroblasts by PPAR $\gamma 2$, a lipid-activated transcription factor. Cell 79, 1147-1156 (1994).

27. Akiyama, H., Chaboissier, M.-C., Martin, J. F., Schedl, A. \& de Crombrugghe, B. The transcription factor Sox9 has essential roles in successive steps of the chondrocyte differentiation pathway and is required for expression of Sox 5 and Sox6. Genes Dev. 16, 2813-2828 (2002).

28. Bi, W., Deng, J. M., Zhang, Z., Behringer, R. R. \& de Crombrugghe, B. Sox9 is required for cartilage formation. Nat. Genet. 22, 85-89 (1999).

29. Rau, M. J., Fischer, S. \& Neumann, C. J. Zebrafish Trap230/Med12 is required as a coactivator for Sox9-dependent neural crest, cartilage and ear development. Dev. Biol. 296, 83-93 (2006).

30. Takarada, T. et al. An analysis of skeletal development in osteoblast-specific and chondrocyte-specific runt-related transcription factor-2 (Runx2) knockout mice. J. Bone Mineral Res. 28, 2064-2069 (2013). 
31. Chen, H. et al. Runx2 regulates endochondral ossification through control of chondrocyte proliferation and differentiation. J. Bone Mineral Res. 29, 2653-2665 (2014).

32. Lee, B. et al. Missense mutations abolishing DNA binding of the osteoblast-specific transcription factor OSF2/CBFA1 in cleidocranial dysplasia. Nat. Genet. 16, 307-310 (1997).

33. Otto, F., Kanegane, H. \& Mundlos, S. Mutations in the RUNX2 gene in patients with cleidocranial dysplasia. Hum. Mutat. 19, 209-216 (2002).

34. Lou, Y. et al. A Runx2 threshold for the cleidocranial dysplasia phenotype. Hum. Mol. Genet. 18, 556-568 (2009).

35. Dobreva, G. et al. SATB2 is a multifunctional determinant of craniofacial patterning and osteoblast differentiation. Cell 125, 971-986 (2006).

36. Nishikawa, K. et al. Maf promotes osteoblast differentiation in mice by mediating the age-related switch in mesenchymal cell differentiation. J. Clin. Invest. 120, 3455-3465 (2010).

\section{Acknowledgements}

We thank Dr Henry M. Kronenberg (Harvard, Massachusetts General Hospital), Dr Yingzi Yang (Harvard, School of Dental Medicine) and Dr Yi-ping Li (the University of Alabama at Birmingham) for suggestions; $\mathrm{Yu}$ Fu and Ke Li for experimental animal breeding and technical assistance. This work was supported in part by grants from the National Natural Science Foundation of China (NSFC; 3127145231371463 and 91519311), the Chinese Academy of Sciences (CAS; XDA01010401), 973 Program the Chinese Ministry of Science and Technology (MOST; 2014CB964702 and 2014CB964704) and 'the National Science Fund for Excellent Young Scholars' (NSFC 81322027). W.Z. is a scholar of the 'National 1000 Young Talents Program of China'.

\section{Author contributions}

Z.L. performed the majority of experiments and prepared the manuscript. G.Y. performed in situ hybridization experiments. X.Y., Y.X. and J.Y. analysed RNA-seq data. W.Z. and G.W. designed and supervised the project.

\section{Additional information}

Accession codes: RNA-seq data has been deposited in the Gene Expression Omnibus (GEO) Data Bank (http://www.ncbi.nlm.nih.gov/gen/) under accession code ID GSE 77007.

Supplementary Information accompanies this paper at http://www.nature.com/ naturecommunications

Competing financial interests: The authors declare no competing financial interests.

Reprints and permission information is available online at http://npg.nature.com/ reprintsandpermissions/

How to cite this article: Liu, Z. et al. Mediator MED23 cooperates with RUNX2 to drive osteoblast differentiation and bone development. Nat. Commun. 7:11149 doi: 10.1038/ncomms11149 (2016).

\section{(c) (i)}

This work is licensed under a Creative Commons Attribution 4.0 International License. The images or other third party material in this article are included in the article's Creative Commons license, unless indicated otherwise in the credit line; if the material is not included under the Creative Commons license, users will need to obtain permission from the license holder to reproduce the material. To view a copy of this license, visit http://creativecommons.org/licenses/by/4.0/ 